\title{
BCLC Stage C Adult Hepatocellular Carcinoma
}

National Cancer Institute

\section{Source}

National Cancer Institute. BCLC Stage C Adult Hepatocellular Carcinoma. NCI Thesaurus.

Code C115143.

Advanced hepatocellular carcinoma. Patients may receive new agents in the setting of randomized controlled trials. (HPB (Oxford) 2005; 7(1):35-41) 